\title{
Urinary Markers of Intrarenal Renin-Angiotensin System Activity In Vivo
}

\author{
Lodi C. W. Roksnoer • Koen Verdonk • \\ Anton H. van den Meiracker • Ewout J. Hoorn • \\ Robert Zietse • A. H. Jan Danser
}

Published online: 8 January 2013

(C) Springer Science+Business Media New York 2012

\begin{abstract}
Recent interest focuses on urinary renin and angiotensinogen as markers of renal renin-angiotensin system activity. Before concluding that these components are independent markers, we need to exclude that their presence in urine, like that of albumin (a protein of comparable size), is due to (disturbed) glomerular filtration. This review critically discusses their filtration, reabsorption and local release. Given the close correlation between urinary angiotensinogen and albumin in human studies, it concludes that, in humans, urinary angiotensinogen is a filtration barrier damage marker with the same predictive power as urinary albumin. In contrast, in animals, tubular angiotensinogen release may occur, although tubulus-specific knockout studies do not support a functional role for such angiotensinogen. Urinary renin levels, relative to albumin, are $>200$-fold higher and unrelated to albumin. This may reflect release of renin from the urinary tract, but could also be attributed to activation of filtered, plasma-derived prorenin and/or incomplete tubular reabsorption.
\end{abstract}

Keywords Renin · Prorenin - Angiotensinogen · Proximal tubule $\cdot$ Collecting duct $\cdot$ Diabetes mellitus $\cdot$ Glomerular filtration · Urine - Albumin · Renin-angiotensin system · RAS $\cdot$ Kidney $\cdot$ Hypertension

L. C. W. Roksnoer K. Verdonk - A. H. van den Meiracker

E. J. Hoorn · R. Zietse $\cdot$ A. H. J. Danser

Division of Pharmacology and Vascular Medicine,

Department of Internal Medicine, Erasmus MC,

Rotterdam, The Netherlands

A. H. J. Danser $(\square)$

Division of Vascular Medicine and Pharmacology,

Department of Internal Medicine, room EE1418b,

Erasmus MC, Dr. Molewaterplein 50,

3015 GE Rotterdam, The Netherlands

e-mail: a.danser@erasmusmc.nl

\section{Introduction}

Renal angiotensin (Ang) II levels are several orders of magnitude higher than circulating Ang II levels, and it is, therefore, generally believed that renal Ang II originates at renal tissue sites. Indeed, making use of infusions of ${ }^{125} \mathrm{I}$ labeled Ang I and II, we were able to show that, despite significant uptake of circulating ${ }^{125}$ I-labeled angiotensin II, $>90 \%$ of renal Ang II could not be explained on the basis of such uptake, and thus truly is derived from local synthesis [1]. Where in the kidneys such generation occurs, and what the effects of locally generated Ang II are, has been discussed elsewhere in detail [2-4]. An obvious question is what determines such generation, i.e., where do the renin, angiotensinogen and angiotensin-converting enzyme (ACE) originate that together generate renal Ang II? A role for nonACE enzymes (e.g., chymase) seems unlikely given the virtual absence of renal Ang II following ACE knockout [5].

Renin is made in the juxtaglomerular apparatus, and released into the interstitial space, from where it may reach the circulation via diffusion across the peritubular capillaries. Proximal tubular fluid, however, also contains renin, suggesting that circulating renin is filtered in the kidney [6]. Unexpectedly, renin expression has also been observed in the principal cells of the collecting duct [7]. Kang et al. suggested that such expression is upregulated in the diabetic kidney, and contributes to the elevated levels of prorenin (the inactive precursor of renin) in patients with diabetic nephropathy [8].

Circulating, liver-derived angiotensinogen diffuses into the interstitium, reaching interstitial fluid levels that are comparable to those in blood [9]. In addition, angiotensinogen mRNA has been demonstrated in the proximal straight tubule, suggesting that angiotensinogen synthesis may also occur locally in the kidney, independently of its synthesis in the liver [4]. 
Circulating ACE plays little, if any, role, and, thus, renal Ang II generation will depend entirely on locally expressed, membrane-bound ACE in the kidney [10]. Indeed, in the human kidney, ACE is abundant in the brush border of the proximal tubule, and, remarkably, usually absent in endothelial cells of any vessel type [11]. Endothelial neoexpression of ACE comes into play in different diseases, e.g., diabetes mellitus and chronic arterial hypertension [11].

Recent interest focuses on the occurrence of both renin and angiotensinogen in urine, as markers of renal renin-angiotensin system (RAS) activity, potentially reflecting the disease state $[12 \cdot, 13]$. If indeed the renal levels of both proteins reflect their production at renal tissue sites, their measurement would be a simple manner to determine whether the renal RAS is upregulated, thus reinforcing the need for treatment with a RAS blocker. However, before drawing this conclusion, we need to be certain that these urinary proteins, like albumin, do not simply reflect breakdown of the glomerular filtration barrier, i.e., that they are kidney- and not plasma-derived. If plasmaderived, their clinical value on top of a much cheaper urinary albumin measurement needs to be proven.

This review summarizes all current findings on urinary angiotensinogen and renin. It will also discuss the presence of prorenin in urine. Given the less abundant literature on urinary ACE, it does not focus on this RAS component. Moreover, it will also not address urinary aldosterone and Ang II, since these peptides are much smaller and thus highly likely to easily filter from the circulation. Indeed, all urinary aldosterone is plasma-derived [12•], and thus urinary aldosterone is a well-established parameter of changes in circulating aldosterone.

\section{Urinary Angiotensinogen}

Animal Studies. Infusing Ang II (40 ng/min) in rats on top of a high-salt diet $(8 \%)$ not only raised the renal Ang II content, but also increased the urinary angiotensinogen excretion 4-fold, from 1 to $4 \mathrm{nmol} /$ day [14]. At a rat urinary volume of $\approx 10 \mathrm{~mL} /$ day, this corresponds with urinary angiotensinogen levels of $100-400 \mathrm{pmol} / \mathrm{mL}$. Given earlier reports on angiotensinogen expression in proximal tubular cells [15], and the fact that in this study urinary angiotensinogen excretion correlated with renal Ang II but not plasma Ang II, it was suggested that urinary angiotensinogen reflects renal Ang II production. Surprisingly, Ang II in fact stimulated renal angiotensinogen synthesis, resulting in both elevated renal angiotensinogen levels and increased urinary angiotensinogen excretion, thus, potentially creating a positive feed-forward loop [16]. According to this concept, the rise in renal Ang II content following Ang II infusion involves de novo Ang II formation in the kidney from locally generated angiotensinogen [17].
When interpreting these results, it should be kept in mind that Ang II also raised blood pressure and is known to stimulate hepatic angiotensinogen production [18]. In combination with the deleterious effects of Ang II on glomerular barrier function, occurring, at least in part, due to the rise in blood pressure, an alternative explanation of these findings is therefore that they reflect increased filtration of circulating, liver-derived angiotensinogen, particularly when its levels are increased [19॰]. Moreover, infusing ${ }^{125}$ I-labeled Ang II results, via Ang II type $1\left(\mathrm{AT}_{1}\right)$ receptor-mediated endocytosis $[20,21]$, in renal ${ }^{125}$ I-Ang II levels that, per gram wet weight, are $\approx 4-5$-fold higher than the plasma ${ }^{125} \mathrm{I}$ Ang II levels per $\mathrm{mL}$ at steady state [22]. Thus, the rise in renal Ang II following Ang II infusion in rats could be easily attributed to $\mathrm{AT}_{1}$ receptor-mediated endocytosis of infused Ang II.

Interestingly, under high-salt conditions, male rats displayed a higher rise in urinary angiotensinogen excretion than female rats during Ang II infusion [23]. Although this could be attributed to the higher renal angiotensinogen mRNA expression in males exposed to Ang II and high salt, an alternative explanation is the higher degree of proteinuria in the male rats of this study, which would be accompanied by increased filtration of circulating angiotensinogen. The urinary angiotensinogen levels in this study ranged from $\leq 0.1$ (control) to $>30 \mathrm{ng} /$ day (after Ang II infusion) per gram body weight. At a body weight of $\approx 250 \mathrm{~g}$ and a urinary volume of $\approx 10 \mathrm{~mL} /$ day, this would correspond with urinary angiotensinogen concentrations of $2.5-750 \mathrm{ng} / \mathrm{mL}(0.04-$ $12 \mathrm{pmol} / \mathrm{mL}$ ). Thus, the control angiotensinogen levels in this study are $>1000$-fold lower than those in the above described studies.

Rats exposed to deoxycorticosterone acetate plus high salt display hypertension but not elevated Ang II levels. In this model, urinary angiotensinogen remained in the normal range $(1 \mathrm{nmol} /$ day) [24]. Yet, the Dahl salt-sensitive rat, when put on a high-salt diet (suppressing the circulating RAS) did display inappropriately elevated renal and urinary angiotensinogen levels in the face of hypertension [25]. Other models evaluating renal dysfunction in the presence of low-to-normal circulating RAS activity (e.g., mice with diabetic nephropathy [26] or IgA nephritis [27] and spontaneously hypertensive rats [28]) also displayed elevated urinary angiotensinogen levels. This could be suggestive for an activated renal RAS, as is believed to exist in diabetes mellitus [29]. If, however, this involves increased renal angiotensinogen expression and the subsequent release of this angiotensinogen into urine, the question is whether the increased renal angiotensinogen expression is the cause or consequence (feed-forward concept) of the elevated renal Ang II levels. The urinary angiotensinogen excretion in diabetic mice amounted to $250 \mu \mathrm{g} /$ day (vs. $20 \mu \mathrm{g}$ /day in controls) at three days after the induction of diabetes. 
Interestingly, in that same time period, while on insulin treatment, the urinary volume also increased $\approx 10$-fold (from $1-1.5$ to $10-15 \mathrm{~mL} /$ day), implying that the actual urinary angiotensinogen concentration did not change. The urinary angiotensinogen concentration in $\operatorname{IgA}$ nephritic mice was $15 \mathrm{ng} / \mathrm{mL}$, threefold higher than in control mice. Assuming a mouse urinary volume of $\approx 1 \mathrm{~mL} /$ day, this would imply that these mice excreted $15 \mathrm{ng}$ angiotensinogen/day (vs. $5 \mathrm{ng} /$ day in controls), i.e., $>1000$-fold less than the mice of the diabetes study.

Taken together, the current animal studies report a wide range ( $>1000$-fold) of urinary angiotensinogen concentrations, even in normal controls, and these levels are, confusingly, presented either per animal per day, per g body weight per day or per $\mathrm{mL}$, and in either grams or moles. Only rarely, plasma and urinary angiotensinogen levels have simultaneously been determined [27]. Unfortunately, the plasma levels were often reported in densitometric units on the basis of Western blot analysis [25], thus not allowing a comparison with the actual urinary levels of angiotensinogen. This raises the need for a reference protein in urine, preferably of identical size. An obvious candidate is albumin, which has a comparable molecular weight ( $67 \mathrm{kD}$ vs. $65 \mathrm{kD})$, and is a well-established marker of nephropathy. Since urinary albumin is generally believed to be entirely plasma-derived, a careful comparison of the urinary albumin and angiotensinogen levels would clarify to what degree urinary angiotensinogen is plasma-derived as well.

Human Studies. Unfortunately, data on the urinary albumin levels in the above animal studies are scarce. Yet, ample studies have evaluated the two proteins in urine in humans. In fact, in these studies, without exception, a strong correlation was observed between urinary angiotensinogen and albumin $[12 \cdot, 13,30,31]$. To study angiotensinogen independently of this relation, Saito et al. selected 28 type 1 diabetes mellitus patients without microalbuminuria, and still observed a 3-fold rise (from 4 to $12 \mu \mathrm{g}$ angiotensinogen/g creatinine) in urinary angiotensinogen versus 21 controls, although this rise was borderline for significance $(P=0.045)$. In type 2 diabetes mellitus patients, urinary angiotensinogen $(10-150 \mu \mathrm{g}$ angiotensinogen $/ \mathrm{g}$ creatinine) correlated strongly with the albumin/creatinine ratio, and predicted the annual decline in the estimated glomerular filtration rate (eGFR) over a 10-year period. Not surprisingly, the patients with both albuminuria and high urinary angiotensinogen levels showed the worst decline of eGFR, and a higher incidence of renal and cardiovascular composite endpoints [30]. Unfortunately, no rigorous statistical analysis was performed to determine the independency of the effect of angiotensinogen and albumin on eGFR decline. Such an analysis was performed in 201 patients with chronic kidney disease (eGFR $<60 \mathrm{~mL} / \mathrm{min} / 1.73 \mathrm{~m}^{2}$ or presence of albuminuria at $\geq 30 \mathrm{mg} / 24 \mathrm{hr}$ ) [31]. Their urinary angiotensinogen levels were 6-fold elevated vs. 201 controls ( 26.3 vs. $4.4 \mu \mathrm{g} / \mathrm{g}$ creatinine), and correlated highly significantly with urinary albumin. Moreover, both urinary angiotensinogen and urinary albumin correlated negatively with eGFR. However, when correcting for albumin, the angiotensinogen-eGFR association remained significant. Thus, elevated urinary angiotensinogen levels on top of elevated urinary albumin levels independently determined eGFR decline. Yet, the analysis did not correct for the elevated plasma angiotensinogen levels that were observed in the patients with chronic kidney disease of this study, and thus it cannot be excluded that the independent effect of urinary angiotensinogen in reality, via glomerular filtration, reflects the activation of the systemic RAS. Yamamoto et al. [32] confirmed the association between urinary angiotensinogen and eGFR decline in patients with chronic kidney disease, and additionally showed that the $\mathrm{AT}_{1}$ receptor antagonist losartan reduced urinary angiotensinogen. Importantly, losartan also reduced plasma angiotensinogen, albeit to a lesser degree. Unfortunately, no comparison with urinary albumin was made in this study.

In hypertensive patients with a preserved kidney function, the urinary angiotensinogen/creatinine ratio correlated with blood pressure and the urinary albumin/creatine ratio [13]. Treatment with RAS blockers decreased the urinary angiotensinogen/creatinine ratio in these patients to levels that were also observed in normotensive controls (from 25 to $14 \mu \mathrm{g} / \mathrm{g}$ creatinine). This decrease was comparable to that in urinary albumin (from 132 to $29 \mathrm{mg} / \mathrm{g}$ creatinine). A correlation between blood pressure and the urinary angiotensinogen/creatinine ratio was even observed after excluding patients with diabetes mellitus or patients who were receiving antihypertensive treatment, in the Bogalusa Heart Study [33]. Lantelme et al. [34] observed a relationship between urinary angiotensinogen and blood pressure only in women with essential hypertension and a low plasma renin/aldosterone ratio, and not in men with this type of hypertension, nor in patients with other types of essential hypertension.

Elevated urinary angiotensinogen/creatinine ratios have been observed in IgA nephropathy in several studies [35, 36], and kidney biopsies of such patients confirmed upregulated expression of angiotensinogen and enhanced Ang II immunoreactivity [36]. Treatment of patients with IgA nephropathy with an $\mathrm{AT}_{1}$ receptor antagonist (valsartan) reduced urinary angiotensinogen [36]. Furthermore, the sodium sensitivity index (i.e., the reciprocal of the slope of the pressure-natriuresis curve drawn by linking the datapoints obtained during a normal- and a low-salt diet) in IgA nephropathy patients correlated positively with the logtransformed urinary angiotensinogen/creatinine ratio (17.5 vs. $7.9 \mu \mathrm{g} / \mathrm{g}$, normal vs. low-salt), but not with the urinary protein 
excretion [37]. However, when analyzing the data, protein excretion was neither corrected for creatinine, nor analyzed with a non-parametric approach despite its usual non-normal distribution, thus making a true comparison with angiotensinogen difficult.

Summarizing, the variation in urinary angiotensinogen levels in humans is much smaller than in rodents, and the levels in healthy individuals are in a very close range: usually $<10 \mu \mathrm{g} / \mathrm{g}$ creatinine, or, at a creatinine level of $\approx 1 \mathrm{~g} / \mathrm{L}, 10 \mathrm{ng}$ angiotensinogen $/ \mathrm{mL}$, i.e., $0.2 \mathrm{pmol} / \mathrm{mL}$. This is several orders of magnitude lower than the urinary levels reported in rodents. Human values were in most cases logtransformed prior to analysis. Studies that also determined urinary albumin or protein usually found a close correlation between these parameters and urinary angiotensinogen, including the changes that occurred following RAS blockade. In the rare cases that the associations between urinary angiotensinogen and disease parameters were independent of or different from albumin (or protein), either the albumin (or protein) data were differently expressed (i.e., not corrected for creatinine and non-log-transformed [37]) or the changes in circulating angiotensinogen were not taken into consideration [31]. The latter is of the utmost importance, to rule out that urinary angiotensinogen truly behaves independently of circulating angiotensinogen. Indeed, when correcting urinary angiotensinogen for plasma angiotensinogen in 101 hypertensive patients with or without diabetes mellitus, we observed that urinary angiotensinogen in all aspects mimicked urinary albumin $[12 \bullet]$.

Filtration Versus Local Synthesis. An elegant study in mice by Nakano et al. has recently investigated to what degree infused human angiotensinogen filters into urine, simultaneously making a comparison with albumin [38・•]. Both proteins were fluorescently labeled and applied via bolus injection into the carotid artery. Immediately thereafter, fluorescence was measured in Bowman's space and the glomerular capillaries to determine glomerular permeability. The data revealed that almost no labeled human angiotensinogen showed up in Bowman's space (four times less than labeled albumin), and that its urinary levels were below the detection limit. Increased sieving of angiotensinogen did occur with an increasing level of glomerulosclerosis, but not to a degree that would explain the rise in urinary angiotensinogen in this condition. Here it should be acknowledged that both Richoux et al. and Pohl et al. [19•, 39] have demonstrated that angiotensinogen does occur in the ultrafiltrate, but is largely removed via endocytotic uptake in the tubulus, in a megalin-dependent manner. Endocytosed angiotensinogen is subsequently degraded, and the amount of angiotensinogen in the proximal convoluted tubules correlated closely with the plasma level of angiotensinogen [39].
Importantly, in the Nakano et al. study [38••], the glomerular permeability of infused human angiotensinogen correlated with the urinary albumin levels, but not with the urinary rodent angiotensinogen levels. The authors therefore proposed that endogenous urinary angiotensinogen originated in the kidney, most likely in the tubules. They detected urinary mouse angiotensinogen levels of $\approx 2 \mathrm{ng} / 90$ minutes versus $\approx 2000 \mathrm{ng} / \mathrm{mL}$ in plasma. In rats developing glomerulosclerosis, the urinary angiotensinogen levels were $\approx 30 \mathrm{ng} / 90$ minutes at young age, and these levels increased to $\approx 100 \mathrm{ng} / 90$ minutes at older age. Given the urinary volume of rodents of at most a few $\mathrm{mL}$ per day, this implies that in these animals the endogenous angiotensinogen levels in urine, like in all previous rodent studies, were several orders of magnitude above those in humans.

When considering the absence of human angiotensinogen in rodent urine in the above study, it should be taken into account that the application of a bolus injection will not result in a stable steady-state level of angiotensinogen in blood. Nakano et al. measured circulating angiotensinogen at one time point (90 minutes after injection), and found it to be equal to the level of endogenous mouse angiotensinogen. However, angiotensinogen, like renin, normally diffuses into the interstitium $[9,40]$, reaching interstitial levels that are comparable to those in plasma [41-43]. Consequently, a substantial amount of the infused human angiotensinogen would be expected to diffuse into the interstitial space in the first few hours post-injection, thereby potentially limiting its accumulation in urine. Normal angiotensinogen levels in human urine are $\approx 0.2 \mathrm{pmol} / \mathrm{mL}$ (see above), versus $\approx 1200 \mathrm{pmol} / \mathrm{mL}$ in plasma, i.e., the urinary levels are at most $0.01 \%$ of the plasma levels. It is, therefore, likely that, in mice, the urinary levels of human angiotensinogen, in view of its whole-body distribution, remained $<0.01 \%$ of its post-injection plasma level, i.e., (far) below the detection limit of the applied assay. A similar line of reasoning applies to a study where the authors failed to detect human angiotensinogen in rat urine following its intravenous injection [24].

A unifying concept, given the $>100$-fold higher urinary angiotensinogen levels in rodents, might be that in humans urinary angiotensinogen is exclusively plasma-derived, hence, its close correlation with albumin, whereas in rodents it is indeed largely kidney (proximal tubule)-derived (see Fig. 1). To investigate the physiological importance of such kidney-derived angiotensinogen, a kidney-specific angiotensinogen knockout mouse has been developed [44••]. Unexpectedly, such mice had renal angiotensinogen and Ang II levels that were identical to those in control mice, both under normal conditions and following podocyte injury. Thus, it was concluded that, even if angiotensinogen is synthesized in the kidney, it has no functional role, i.e., it does not contribute to renal angiotensin production. This contradicts the feed-forward loop between Ang II and renal 


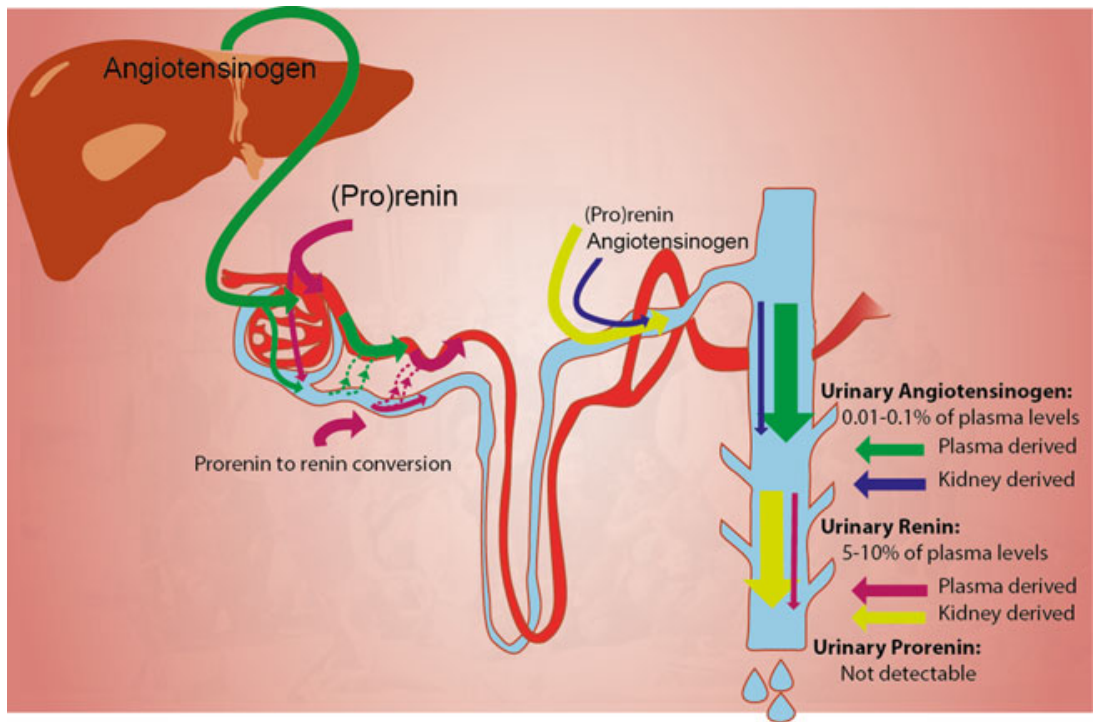

Fig. 1 Overview of the origin of urinary renin and angiotensinogen. In humans, angiotensinogen in urine correlates closely with albumin, and thus, like albumin, reaches urine via glomerular filtration from plasma. Levels range from $<10-150 \mu \mathrm{g} / \mathrm{g}$ creatinine, or (assuming a creatinine content of $\approx 1 \mathrm{~g} / \mathrm{L}$ ),$<10-150 \mathrm{ng} / \mathrm{mL}$ (i.e., $<0.2-2 \mathrm{pmol} / \mathrm{mL}$ ). This corresponds with $<0.01-0.1 \%$ of the plasma angiotensinogen levels in humans. In rodents, urinary angiotensinogen levels are usually much higher, and range from $<0.5-4000 \mathrm{pmol} / \mathrm{day}$, or (assuming a urinary volume of $\approx 1 \mathrm{~mL} /$ day in mice and $\approx 10 \mathrm{~mL} /$ day in rats) $<0.1-400 \mathrm{pmol} / \mathrm{mL}$. This implies that the urinary angiotensinogen

angiotensinogen [16], but would still imply that (non-functional) angiotensinogen in the urine of rodents provides an indication of the amount of Ang II to which the kidney (proximal tubule) is exposed. Clearly, evidence is now needed to what degree this holds true in animal models with severe glomerular damage, resulting in increased filtration of plasma proteins, including albumin and angiotensinogen.

\section{Urinary Renin and Prorenin}

Renin was originally described in human urine by Lumbers and Skinner $[45,46]$. They found no relationship with urinary creatinine or urinary protein, nor with plasma renin. On average, the urinary renin level was $\approx 7 \%$ of that in plasma. Remarkably, although women normally have lower plasma renin levels than men [47], their urinary renin levels were higher. Natriuretic therapy (spironolactone + chlorothiazide) elevated plasma renin 5-fold, but only modestly increased urinary renin. As a consequence, renin clearance $(\mathrm{mL}$ plas$\mathrm{ma} / 24$ hours) fell by $>50 \%$, whereas protein clearance fell by $\approx 20 \%$. The authors concluded that during sodium depletion either increased tubular reabsorption of renin had occurred, or that urinary renin originated from the urinary tract (tubular sites and/or the collecting duct). In case of the latter, such local renin release then apparently remained relatively constant in the face of increased circulating renin levels. levels in rodents sometimes are higher than their plasma levels. Such high levels could reflect angiotensinogen release from renal tissue sites, possibly the proximal tubule. Urinary renin levels in humans were $5-10 \%$ of the plasma renin levels, i.e., 1-2 orders of magnitude higher than the corresponding urinary levels of albumin and angiotensinogen. Prorenin was undetectable in urine. Most likely, therefore, urinary renin has 3 sources: filtration from plasma, release from the urinary tract (possibly the collecting duct), and intrarenal conversion of (plasma-derived) prorenin to renin

Yukimura et al. confirmed the modest role of urinary excretion in the metabolism of renin, and were unable to demonstrate prorenin in the urine of dogs [48]. Unlike Lumbers and Skinner, these authors did see a rise in urinary renin excretion when elevating plasma renin in dogs, although in this case plasma renin was elevated by injecting semipurified kidney renin. Mazanti et al. found that renin in mouse urine was two orders of magnitude lower than in plasma, and also observed an increase in urinary renin following injection of exogenous renin [49]. Importantly, when blocking tubular reabsorption with lysine, urinary renin rose $>100$-fold, without altering plasma renin [49]. This suggests that normally, ultrafiltered renin is reabsorbed almost completely. Indeed, Pohl et al. confirmed such reabsorption, and suggested that it involves megalin, since immunoreactive renin could not be demonstrated in the proximal tubulus of mice lacking tubular megalin [19•]. Nielsen et al. observed very little, if any, prorenin in mouse urine [50]. However, after lysine, prorenin levels in urine became detectable, and the authors were able to estimate that the urinary clearance of prorenin in the absence of tubular reabsorption was $\approx 10$-fold lower than that of renin.

In a recent study involving 101 diabetic and non-diabetic patients with or without hypertension, we observed urinary renin levels that were around $\approx 6 \%$ of those in plasma [12•], identical to the observations by Lumbers and Skinner [45, 46]. Prorenin was undetectable in urine in our hands, and 
this was not due to prorenin-renin conversion in urine, since adding prorenin to urine did not result in renin generation. Renin in urine was unrelated to urinary albumin or angiotensinogen, and relative to these proteins $>200$-fold higher. Urinary renin did not correlate with plasma renin or prorenin. Unexpectedly, urinary renin decreased in patients treated with a RAS blocker (despite their elevated plasma renin levels), and increased in diabetics (despite their lower plasma renin levels). This would be in agreement with the concept that urinary renin is derived from the distal nephron, i.e., the collecting duct, and that Ang II stimulates the release of renin/prorenin from this site, as opposed to its inhibitory role towards renin release from the juxtaglomerular cells. Indeed, several animal studies support the concept of renin synthesis in the principal cells of the collecting duct, and its upregulation by Ang II and diabetes mellitus [7, 8, 51].

The inability to detect prorenin in human urine may be so for several reasons. First, concentrations may be too low to detect, implying that prorenin is not filtered to the same degree as renin, reabsorbed to a greater degree, and/or not released from the collecting duct. Second, proteolytic cleavage of filtered prorenin may have occurred in the kidney, during the formation of urine. Clearly, such cleavage does not occur in urine per se (given our inability to observe prorenin-renin conversion when adding prorenin to urine ex vivo), although at the same time it is known that the kidneys are the only sites in the body where prorenin-renin conversion occurs $[52,53]$.

In summary, it appears that circulating renin is filtered in the glomerulus (given its presence in proximal tubular fluid [54]), but subsequently highly efficiently reabsorbed in the proximal tubule (see Fig. 1). This resembles the renal handling of circulating angiotensinogen [19•]. For unknown reasons, filtration of circulating prorenin tends to occur to a lesser degree, and is likely to be followed by proteolytic cleavage to renin in the urinary system, thus explaining why urinary prorenin is undetectable in most studies. In addition, there may be Ang II-stimulated renin release from the collecting duct. This would explain why urinary renin levels are far above those of albumin and angiotensinogen, and do not run in parallel with changes in plasma renin.

\section{Conclusion}

In humans, urinary angiotensinogen correlates closely with urinary albumin, and when carefully correcting for changes in circulating angiotensinogen, there is as yet no convincing evidence that there are conditions where urinary angiotensinogen behaves independently of urinary albumin. Therefore, urinary angiotensinogen, like albumin, clearly is a marker of renal disease, and the measurement of both proteins offers the same information. Interestingly, using a proteomics approach, Alge et al. recently identified urinary angiotensinogen and albumin as the two strongest biomarkers in patients with acute kidney injury predicting the need for renal replacement therapy [55]. These investigators subsequently confirmed the prognostic predictive power of urinary angiotensinogen in 97 patients who underwent cardiac surgery for the development of acute kidney injury. Unfortunately, they did not verify the prognostic power of urinary albumin, despite the many studies that support such a role [56-58].

In rodents, there may be a release of non-functional angiotensinogen into urine from proximal tubular synthesis sites. Ang II stimulates such release. Remarkably, this angiotensinogen neither contributes to the renal angiotensinogen content nor to the renal Ang II levels [44••], implying that all functionally renal angiotensinogen is liver-derived. Liver-derived angiotensinogen may reach renal tissue sites via diffusion into the interstitium and/or glomerular filtration.

Urinary renin potentially has three sources: filtration from plasma, release from the collecting duct, and intrarenal conversion of (plasma-derived) prorenin to renin. Normally, filtered renin is highly efficiently reabsorbed, and the same applies to filtered prorenin. Thus, most urinary renin would then be derived from the collecting duct. Only when greatly elevating circulating renin (e.g., by infusing exogenous renin or by blocking the RAS highly efficiently), tubular reabsorption falls short, allowing plasma renin to also accumulate in urine. The same will occur when plasma prorenin levels are elevated, provided that filtered prorenin is converted to renin in the urinary system. Thus, an alternative explanation for the elevated urinary renin levels in diabetics vs. non-diabetics is that it is the consequence of their elevated prorenin levels $[12 \bullet, 47]$. Clearly, we need prospective studies evaluating the use of urinary renin as an independent marker, e.g., to establish the renal efficacy of RAS blockers and renal RAS activity in diseases such as diabetes mellitus and CKD.

Acknowledgment E.J. Hoorn is supported by a grant from The Netherlands Organisation for Scientific Research (NWO, Veni grant, 91612140).

Disclosure No potential conflicts of interest relevant to this article were reported.

\section{References}

Recently published papers of particular interest have been highlighted as:

- Of importance

•• Of major importance

1. van Kats JP, Schalekamp MADH, Verdouw PD, et al. Intrarenal angiotensin II: interstitial and cellular levels and site of production. Kidney Int. 2001;60:2311-7. 
2. Schalekamp MADH, Danser AHJ. Angiotensin II production and distribution in the kidney: I. A kinetic model. Kidney Int. 2006;69:1543-52.

3. Schalekamp MADH, Danser AHJ. Angiotensin II production and distribution in the kidney: II. Model-based analysis of experimental data. Kidney Int. 2006;69:1553-7.

4. Kobori H, Nangaku M, Navar LG, Nishiyama A. The intrarenal renin-angiotensin system: from physiology to the pathobiology of hypertension and kidney disease. Pharmacol Rev. 2007;59: 251-87.

5. Alexiou T, Boon WM, Denton DA, et al. Angiotensinogen and angiotensin-converting enzyme gene copy number and angiotensin and bradykinin peptide levels in mice. J Hypertens. 2005;23: 945-54.

6. Leyssac PP. Changes in single nephron renin release are mediated by tubular fluid flow rate. Kidney Int. 1986;30:332-9.

7. Prieto-Carrasquero MC, Harrison-Bernard LM, Kobori H, et al. Enhancement of collecting duct renin in angiotensin II-dependent hypertensive rats. Hypertension. 2004;44:223-9.

8. Kang JJ, Toma I, Sipos A, et al. The collecting duct is the major source of prorenin in diabetes. Hypertension. 2008;51:1597-604.

9. de Lannoy LM, Danser AHJ, van Kats JP, et al. Renin-angiotensin system components in the interstitial fluid of the isolated perfused rat heart. Local production of angiotensin I. Hypertension. 1997;29:1240-51.

10. Danser AHJ, Batenburg WW, van den Meiracker AH, Danilov SM. ACE phenotyping as a first step toward personalized medicine for ACE inhibitors. Why does ACE genotyping not predict the therapeutic efficacy of ACE inhibition? Pharmacol Ther. 2007;113:607-18.

11. Metzger R, Bohle RM, Pauls K, et al. Angiotensin-converting enzyme in non-neoplastic kidney diseases. Kidney Int. 1999; $56: 1442-54$.

12. - van den Heuvel M, Batenburg WW, Jainandunsing S, et al. Urinary renin, but not angiotensinogen or aldosterone, reflects the renal renin-angiotensin-aldosterone system activity and the efficacy of renin-angiotensin-aldosterone system blockade in the kidney. J Hypertens. 2011;29:2147-55. This paper is the first to simultaneously analyze angiotensinogen and albumin in plasma and urine of diabetic and hypertensive patients.

13. Kobori H, Alper Jr AB, Shenava R, et al. Urinary angiotensinogen as a novel biomarker of the intrarenal renin-angiotensin system status in hypertensive patients. Hypertension. 2009;53:344-50.

14. Kobori H, Harrison-Bernard LM, Navar LG. Urinary excretion of angiotensinogen reflects intrarenal angiotensinogen production. Kidney Int. 2002;61:579-85.

15. Rohrwasser A, Morgan T, Dillon HF, et al. Elements of a paracrine tubular renin-angiotensin system along the entire nephron. Hypertension. 1999;34:1265-74.

16. Kobori H, Prieto-Carrasquero MC, Ozawa Y, Navar LG. AT1 receptor mediated augmentation of intrarenal angiotensinogen in angiotensin II-dependent hypertension. Hypertension. 2004;43:1126-32.

17. Gonzalez-Villalobos RA, Satou R, Seth DM, et al. Angiotensinconverting enzyme-derived angiotensin II formation during angiotensin II-induced hypertension. Hypertension. 2009;53:351-5.

18. Herrmann HC, Dzau VJ. The feedback regulation of angiotensinogen production by components of the renin-angiotensin system. Circ Res. 1983;52:328-34.

19. • Pohl M, Kaminski H, Castrop H, et al. Intrarenal renin angiotensin system revisited: role of megalin-dependent endocytosis along the proximal nephron. J Biol Chem. 2010;285:41935-46. This paper provides a detailed analysis of the reabsorption of RAS components in the kidney.

20. van Kats JP, van Meegen JR, Verdouw PD, et al. Subcellular localization of angiotensin II in kidney and adrenal. J Hypertens. 2001;19:583-9.
21. Zou LX, Imig JD, von Thun AM, et al. Receptor-mediated intrarenal angiotensin II augmentation in angiotensin II-infused rats. Hypertension. 1996;28:669-77.

22. van Kats JP, de Lannoy LM, Danser AHJ, et al. Angiotensin II type 1 (AT1) receptor-mediated accumulation of angiotensin II in tissues and its intracellular half-life in vivo. Hypertension. 1997; 30:42-9.

23. Rands VF, Seth DM, Kobori H, Prieto MC. Sexual dimorphism in urinary angiotensinogen excretion during chronic angiotensin IIsalt hypertension. Gend Med. 2012;9:207-18.

24. Kobori H, Nishiyama A, Harrison-Bernard LM, Navar LG. Urinary angiotensinogen as an indicator of intrarenal angiotensin status in hypertension. Hypertension. 2003;41:42-9.

25. Kobori H, Nishiyama A, Abe Y, Navar LG. Enhancement of intrarenal angiotensinogen in Dahl salt-sensitive rats on high salt diet. Hypertension. 2003;41:592-7.

26. Kamiyama M, Zsombok A, Kobori H. Urinary angiotensinogen as a novel early biomarker of intrarenal renin-angiotensin system activation in experimental type 1 diabetes. J Pharmacol Sci. 2012;119:314-23.

27. Kobori H, Katsurada A, Miyata K, et al. Determination of plasma and urinary angiotensinogen levels in rodents by newly developed ELISA. Am J Physiol Renal Physiol. 2008;294:F1257-63.

28. Susic D, Frohlich ED, Kobori H, et al. Salt-induced renal injury in SHRs is mediated by AT1 receptor activation. J Hypertens. 2011;29:716-73.

29. Hollenberg NK, Fisher ND, Nussberger J, et al. Renal responses to three types of renin-angiotensin system blockers in patients with diabetes mellitus on a high-salt diet: a need for higher doses in diabetic patients? J Hypertens. 2011;29:2454-61.

30. Sawaguchi M, Araki SI, Kobori H, et al. Association between urinary angiotensinogen levels and renal and cardiovascular prognoses in patients with type 2 diabetes mellitus. J Diabetes Investig. 2012;3:318-24.

31. Mills KT, Kobori H, Hamm LL, et al. Increased urinary excretion of angiotensinogen is associated with risk of chronic kidney disease. Nephrol Dial Transplant. 2012;27:3176-81.

32. Yamamoto T, Nakagawa T, Suzuki H, et al. Urinary angiotensinogen as a marker of intrarenal angiotensin II activity associated with deterioration of renal function in patients with chronic kidney disease. J Am Soc Nephrol. 2007;18:1558-65.

33. Kobori $\mathrm{H}$, Urushihara $\mathrm{M}, \mathrm{Xu} \mathrm{JH}$, et al. Urinary angiotensinogen is correlated with blood pressure in men (Bogalusa Heart Study). J Hypertens. 2010;28:1422-8.

34. Lantelme P, Rohrwasser A, Vincent M, et al. Significance of urinary angiotensinogen in essential hypertension as a function of plasma renin and aldosterone status. J Hypertens. 2005; 23:785-92.

35. Jang HR, Kim SM, Lee YJ, et al. The origin and the clinical significance of urinary angiotensinogen in proteinuric IgA nephropathy patients. Ann Med. 2012;44:448-57.

36. Nishiyama A, Konishi Y, Ohashi N, et al. Urinary angiotensinogen reflects the activity of intrarenal renin-angiotensin system in patients with IgA nephropathy. Nephrol Dial Transplant. 2011; 26:170-7.

37. Konishi Y, Nishiyama A, Morikawa T, et al. Relationship between urinary angiotensinogen and salt sensitivity of blood pressure in patients with IgA nephropathy. Hypertension. 2011;58:205-11.

38. • Nakano D, Kobori H, Burford JL, et al. Multiphoton imaging of the glomerular permeability of angiotensinogen. J Am Soc Nephrol. 2012;23:1847-56. This paper compares the glomerular filtration of angiotensinogen and albumin in vivo under normal and pathological conditions.

39. Richoux JP, Cordonnier JL, Bouhnik J, et al. Immunocytochemical localization of angiotensinogen in rat liver and kidney. Cell Tissue Res. 1983;233:439-51. 
40. Hirsch AT, Opsahl JA, Lunzer MM, Katz SA. Active renin and angiotensinogen in cardiac interstitial fluid after myocardial infarction. Am J Physiol. 1999;276:H1818-26.

41. van Kats JP, Danser AHJ, van Meegen JR, et al. Angiotensin production by the heart: a quantitative study in pigs with the use of radiolabeled angiotensin infusions. Circulation. 1998;98:73-81.

42. Danser AHJ, van Kesteren CAM, Bax WA, et al. Prorenin, renin, angiotensinogen, and angiotensin-converting enzyme in normal and failing human hearts. Evidence for renin binding. Circulation. 1997;96:220-6.

43. Klotz S, Burkhoff D, Garrelds IM, et al. The impact of left ventricular assist device-induced left ventricular unloading on the myocardial renin-angiotensin-aldosterone system: therapeutic consequences? Eur Heart J. 2009;30:805-12.

44. •- Matsusaka T, Niimura F, Shimizu A, et al. Liver angiotensinogen is the primary source of renal angiotensin II. J Am Soc Nephrol. 2012;23:1181-9. Using a kidney-specific knockout approach, this paper demonstrates that renal angiotensin generation depends on hepatic angiotensinogen.

45. Lumbers ER, Skinner SL. Observations on the origin of renin in human urine. Circ Res. 1969;24:689-97.

46. Lumbers ER, Skinner SL. The occurrence and assay of renin in human urine. Aust J Exp Biol Med Sci. 1969;47:251-62.

47. Danser AHJ, Derkx FHM, Schalekamp MADH, et al. Determinants of interindividual variation of renin and prorenin concentrations: evidence for a sexual dimorphism of (pro)renin levels in humans. J Hypertens. 1998;16:853-62.

48. Yukimura T, Miura K, Matsushima Y, et al. Urinary excretion of renin and its biochemical properties in dogs. Hypertension. $1984 ; 6: 837-42$.
49. Mazanti I, Hermann KL, Nielsen AH, Poulsen K. Ultrafiltration of renin in the mouse kidney studied by inhibition of tubular protein reabsorption with lysine. Clin Sci (Lond). 1988;75:331-6.

50. Nielsen AH, Hermann KL, Mazanti I, Poulsen K. Urinary excretion of inactive renin during blockade of the renal tubular protein reabsorption with lysine. J Hypertens. 1989;7:77-82.

51. Prieto-Carrasquero MC, Kobori H, Ozawa Y, et al. AT1 receptormediated enhancement of collecting duct renin in angiotensin IIdependent hypertensive rats. Am J Physiol Renal Physiol. 2005; 289:F632-7.

52. Krop M, Danser AHJ. Circulating versus tissue renin-angiotensin system: on the origin of (pro)renin. Curr Hypertens Rep. 2008; 10:112-8.

53. Krop M, de Bruyn JHB, Derkx FHM, Danser AHJ. Renin and prorenin disappearance in humans post-nephrectomy: evidence for binding? Front Biosci. 2008;13:3931-9.

54. Leyssac PP. A micropuncture study of glomerular filtration and tubular reabsorption of endogenous renin in the rat. Renal Physiol. 1978;1:181-8.

55. Alge JL, Karakala N, Neely BA, et al. Urinary angiotensinogen and risk of severe AKI. Clin J Am Soc Nephrol. 2012.

56. Hillege HL, Fidler V, Diercks GF, et al. Urinary albumin excretion predicts cardiovascular and noncardiovascular mortality in general population. Circulation. 2002;106:1777-82.

57. Menne J, Chatzikyrkou C, Haller H. Microalbuminuria as a risk factor: the influence of renin-angiotensin system blockade. J Hypertens. 2010;28:1983-94.

58. Jackson CE, Solomon SD, Gerstein HC, et al. Albuminuria in chronic heart failure: prevalence and prognostic importance. Lancet. 2009;374:543-50. 